\title{
Application of artificial neural networks for automated analysis of cystoscopic images: a review of the current status and future prospects
}

\author{
Misgana Negassi ${ }^{1,2} \cdot$ Rodrigo Suarez-lbarrola $^{3} \cdot$ Simon Hein $^{3} \cdot$ Arkadiusz Miernik $^{3} \cdot$ Alexander Reiterer $^{1,2}$
}

Received: 25 July 2019 / Accepted: 13 December 2019 / Published online: 10 January 2020

(c) The Author(s) 2020

\begin{abstract}
Background Optimal detection and surveillance of bladder cancer (BCa) rely primarily on the cystoscopic visualization of bladder lesions. AI-assisted cystoscopy may improve image recognition and accelerate data acquisition.

Objective To provide a comprehensive review of machine learning (ML), deep learning (DL) and convolutional neural network (CNN) applications in cystoscopic image recognition.

Evidence acquisition A detailed search of original articles was performed using the PubMed-MEDLINE database to identify recent English literature relevant to ML, DL and CNN applications in cystoscopic image recognition.

Evidence synthesis In total, two articles and one conference abstract were identified addressing the application of AI methods in cystoscopic image recognition. These investigations showed accuracies exceeding $90 \%$ for tumor detection; however, future work is necessary to incorporate these methods into AI-aided cystoscopy and compared to other tumor visualization tools. Furthermore, we present results from the RaVeNNA-4pi consortium initiative which has extracted 4200 frames from 62 videos, analyzed them with the U-Net network and achieved an average dice score of 0.67 . Improvements in its precision can be achieved by augmenting the video/frame database.

Conclusion AI-aided cystoscopy has the potential to outperform urologists at recognizing and classifying bladder lesions. To ensure their real-life implementation, however, these algorithms require external validation to generalize their results across other data sets.
\end{abstract}

Keywords Neural networks · Deep learning $\cdot$ Cystoscopic images $\cdot$ Medical image analysis

Alexander Reiterer

alexander.reiterer@ipm.fraunhofer.de

Misgana Negassi

misgana.negassi@ipm.fraunhofer.de

Rodrigo Suarez-Ibarrola

rodrigo.suarez@uniklinik-freiburg.de

Simon Hein

simon.hein@uniklinik-freiburg.de

Arkadiusz Miernik

arkadiusz.miernik@uniklinik-freiburg.de

1 Department of Sustainable Systems Engineering INATECH, University of Freiburg, Emmy-Noether-Straße 2, Freiburg, Germany

2 Department Object and Shape Detection, Fraunhofer Institute for Physical Measurement Techniques IPM, Heidenhofstraße 8, Freiburg, Germany

3 Department of Urology, Faculty of Medicine, University of Freiburg-Medical Centre, Hugstetter Str. 55, Freiburg, Germany

\section{Introduction}

Bladder cancer (BCa) accounts for approximately $7 \%$ of all newly diagnosed cancers in the USA, being the fourth most common cancer and eighth most lethal in men $[1,2]$. In Germany, BCa represents $4.7 \%$ of all new cancer cases and $3.2 \%$ of all cancer-related deaths [3]. Despite a low stage and grade, recurrence rates range from 50 to $75 \%$ at 20 years, setting patients at risk of progression to muscle-invasive disease [4]. Periodic cystoscopic examinations remain the cornerstone of patient follow-up to detect early recurrences and reduce the risk of progression [5]. However, cystoscopic findings are diverse and often challenging to recognize and classify, ranging from healthy tissue to urothelial carcinoma [6]. A precise recognition of these features currently depends on the examiner's skill and experience, leading to wide inter-observer variability in the interpretation of cystoscopic findings [7]. 
Artificial intelligence (AI) is becoming more prevalent in the field of medicine as a critical component of computeraided diagnosis (CAD) and subsequent treatment. This has been notably shown in the successful application of deep learning (DL) approaches in various medical image analysis tasks [8]. Such works include image classification, segmentation, detection and registration of data obtained from radiology, pathology and endoscopy. The emergence of AIassisted endoscopy, by training DL algorithms with large data sets of images or videos, has shown promising results, providing CAD tools that improve lesion detection and achieve diagnosis with high sensitivity and specificity $[9$, 10]. AI-assisted endoscopy offers the potential to radically change surgical practice by assisting physicians in identifying areas of malignancy given the heterogeneous aspect of lesions [11]. In the field of urology, cystoscopy, in particular, can benefit from this methodology. The applicability of CAD in cystoscopic examinations, however, has not been extensively evaluated.

This work provides a brief overview of new methods for tumor visualization, current techniques of automated image evaluation and finally discusses novel AI-based methods to automatically evaluate cystoscopic images.

\section{Evidence acquisition}

A comprehensive review of current literature was performed using the PubMed-Medline database up to October 2019 using the term "cystoscopy", combined with one of the following terms: "machine learning", "deep learning" and "convolutional neural network". To capture recent trends in machine learning (ML), deep learning (DL) and convolutional neural network $(\mathrm{CNN})$ applications, the search was limited to articles and abstracts published within the last 5 years, originally published in English. Review articles and editorials were excluded. Publications relevant to the subject and their cited references were retrieved and appraised independently by two authors (M.N. and A.R.). After full text evaluation, data were independently extracted by the authors for further assessment of qualitative and quantitative evidence synthesis. The following information was extracted from each study: name of author, journal and year of publication, AI method, number of participants per study, and outcome prediction accuracy.

\section{State of the art of urological endoscopy}

Although white light cystoscopy (WLC) is the current standard of care for the initial evaluation of $\mathrm{BCa}$, it has several shortcomings, such as the difficulty to detect small or flat lesions, including carcinoma in situ (Cis) [12].
Current data suggest that early recurrence in BCa patients may be the result of undetected lesions during cystoscopy and transurethral resection of bladder tumors (TURB) [13]. The application of advanced optical techniques is becoming more widespread to improve the diagnostic sensitivity of inconspicuous lesions and prevent recurrent non-muscle invasive bladder cancer (NMIBC).

In photodynamic diagnosis (PDD) or fluorescence cystoscopy, an optical imaging agent is instilled preoperatively into the bladder resulting in the accumulation of protoporphyrins in rapidly proliferating cells such as malignant bladder tumors [14]. They are subsequently converted to photoactive porphyrins emitting a red fluorescent light under blue light. Studies have demonstrated that PDD improves Cis detection and reduces recurrences in patients with known or suspected NMIBC thereby also reducing health-care costs [15]. Its role in the surveillance scenario is emerging for use in patients with high-risk tumor lesions [16].

Narrow band imaging (NBI) has been similarly designed to improve $\mathrm{BCa}$ detection compared to WLC. This technology aims to augment the visualization of tumoral blood vessels by light modulation, by narrowing the bandwidth of light output to 415 and $540 \mathrm{~nm}$, which is strongly absorbed by hemoglobin [17].

Confocal laser endomicroscopy (CLE) couples microscopic imaging with a fiber-optic bundle transmitting a $488 \mathrm{~nm}$ laser light, which provides real-time and in vivo histopathologic information [18]. The CLE probe has a $0.85 \mathrm{~mm}$ to $2.6 \mathrm{~mm}$ diameter, $240 \mu \mathrm{m}$ penetration depth and can pass through the working channel of standard cystoscopes [19]. It uses fluorescein as a contrast agent and can obtain a thorough evaluation of tissue structures to distinguish between low- and high-grade BCa. Unfortunately, a small field of view and reduced tissue penetration limit its ability for surveying the entire bladder. Moreover, largescale studies examining the diagnostic accuracy of CLE, including sensitivity and specificity, are lacking.

Furthermore, new techniques and methods are currently under development: optical coherence tomography (OCT) is a non-invasive real-time microscopic imaging technique using a near-infrared wavelength light (890-1300 nm) with a $2 \mathrm{~mm}$ penetration depth that produces an ultrasound-like image [20]. OCT measures changes of backscattered light with an interferometer caused by asymmetric structures within the tissue and can be applied to standard rigid cystoscopy given its $2.7 \mathrm{~mm}$ diameter probe [20]. Huang et al. recently performed a meta-analysis to evaluate the diagnostic accuracy of OCT. The sensitivity and specificity of the included publications ranged from 76 to $100 \%$ and $62 \%$ and $97 \%$, resulting in a pooled sensitivity and specificity of 0.96 and 0.82 , respectively [21]. OCT is limited by a small area of analysis, making the examination of the entire bladder 
impractical; therefore, its use with other ancillary methods is encouraged.

Raman spectroscopy (RS) is an endomicroscopic technology that does not require photoactive substances and can be used to examine molecular components in tissue. RS uses infrared light (785-845 $\mathrm{nm}$ ) to analyze the inelastic photon scattering after its interaction with intramolecular bonds. RS can determine the integrity of bladder wall layers, assess penetration depth and recognize low- or high-grade $\mathrm{BCa}$ [19]. Chen et al. recently applied a fiber-optic Raman sensing probe to evaluate the diagnostic potential of RS in identifying various bladder pathologies ex vivo. The sensitivities and specificities for normal bladder tissue and low-grade and high-grade bladder tumors were $88.5 \%, 95.1 \%$ and $90.3 \%$, and $98 \%, 97.5 \%$ and $96.4 \%$, respectively [22]. Nevertheless, RS has several limitations, such as a restricted field of view, making the screening of the entire bladder surface impractical and should be targeted at suspicious lesions identified by PDD or NBI.

Multiphoton microscopy (MPM) uses a laser-scanning microscope and simultaneously absorbs two near-infrared photons (700-800 $\mathrm{nm}$ ) based on the autofluorescence of cells and extracellular components with intrinsic tissue fluorophores, such as flavin adenine dinucleotide (FAD) and nicotinamide adenine dinucleotide (NADH) to provide information on cellular metabolic activity [23]. Pradère et al. recently assessed an optical multimodal technique on samples of patients suspected of having $\mathrm{BCa}$ and found that it was able to discriminate tumor from healthy tissue and determine the grade of tumors [24]. However, when MPM is used alone, it is limited by its shallow penetration and the difficulty to recognize intranuclear modifications.

As opposed to the previously mentioned optical techniques, which require a human professional for interpretation, AI methods rely on training CNNs with images or videos so it may learn to recognize complex patterns. Although research in this area has increased dramatically in recent years, there is presently no real-time application of AI-assisted cystoscopy in the clinical setting to accurately and reproducibly classify cystoscopic findings.

\section{Artificial neural networks for analysis of images: an overview}

In image analysis, the term object recognition refers to the detection or classification of objects in image or video data. It describes whether or to what extent input data are similar to previously seen objects. Methods for object class recognition are usually based on a learning process. Classical image processing methods such as SIFT [25] determine from a training data set a suitable selection of features that can be used to reliably identify instances of the object class.
In recent years, object recognition methods using deep learning (DL) or artificial neural networks (ANNs) have shown to be superior to classical methods [26]. ANNs have a strong representational power that enables them to learn complex hierarchical representation of images, thus increasingly representing abstract concepts. They are powerful at generalizing to never seen data. This is especially the case when it comes to recognizing a multitude of different objects whose appearance also varies greatly. ANNs are also more robust against variations, e.g., masking, fading of colors, damage or soiling. As early as the end of the 1990s, ANNs were used to solve simple tasks such as handwriting recognition (socalled LeNet) [27]. DL differs from the classical methods for object recognition primarily in that the objects or object classes to be recognized are not described by a set of manually predefined features, but the feature representation of an object is learned during training from the neural network.

\section{Artificial neural networks}

Most current DL approaches are based on a network architecture known as feedforward neural network or multilayer perceptron (MLP) [28, 29]. These networks use the backpropagation method for the actual learning process, which is also referred to as training. Figure 1 shows the architecture of a simple MLP. Each layer of the neural network consists of a set of neurons. The number of input neurons is chosen according to the input data, and the number of output neurons according to the target result; e.g., the input corresponds

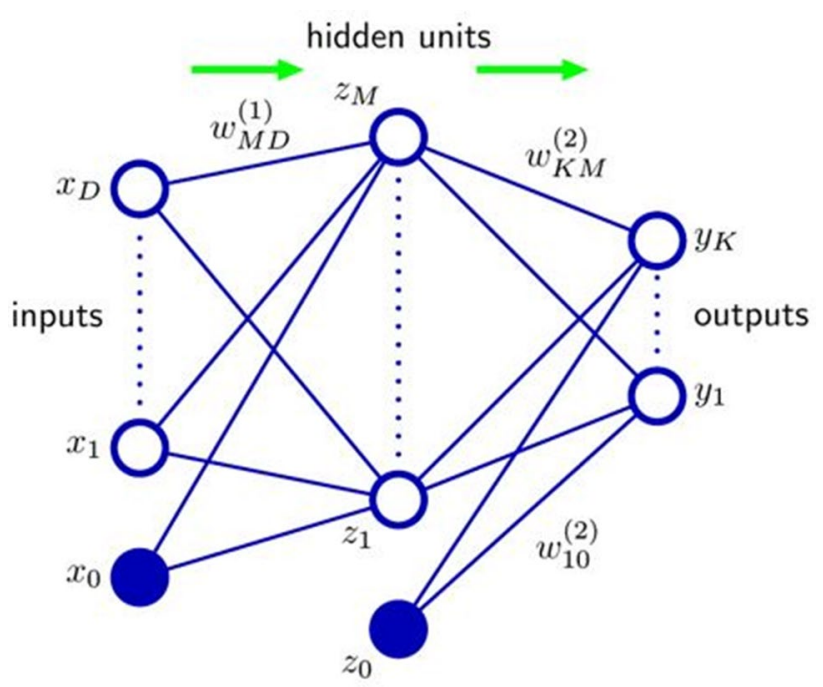

Fig. 1 Multilayer perceptron with input and output layer as well as an additional hidden unit layer. The neurons of a layer are represented as unfilled blue circles, and the completely colored neurons are the socalled bias neurons, whose value is constant and does not depend on the activations in previous layers. The weights of the compounds are learned in training (Source: [1]) 
to the number of pixels in the image, and the output to the number of object classes to be detected. Between the input and the output layer, there are any number of hidden layers, called hidden units in literature. The layers are arranged vertically in Fig. 1; the green arrows symbolize the direction in which the information is passed through the network in a forward propagation. Each neuron is connected to all neurons from the previous layer. The activation of a neuron is thus determined from all activation values in the preceding layer. These are multiplied by the weights of the individual connections to this neuron. The weights are the parameters learned during the training.

The selection of the activation function depends on the application and represents one of the hyperparameters that influence the performance of the neural network. Typical activation functions are, e.g., Rectified Linear Unit (ReLU), Logistic Sigmoid Activation Function or Hyperbolic Tangent (tanh). The activation function must be differentiable so that the derivation or gradient can be determined during training. The activations in subsequent hidden layers are calculated analogously. In summary, the output values are calculated from the input values for the MLP.

Although the MLP seems simple in its structure, many tasks can be solved with it. Thus, any limited continuous function can be approximated with any accuracy by an MLP with only one hidden layer [30]. Based on this simple network structure, a variety of network architectures for different applications have been developed.

\section{Convolutional neural networks}

In the field of image processing, the so-called convolutional neural networks (CNNs) are of particular interest [31]. These networks take advantage of the fact that information in images is mostly local, i.e., limited to a smaller part of the image. They exploit this spatial layout of images by using local connectivity and parameter sharing [32]. Therefore, filters can be used for the analysis of the image content, which only look at a small part of the image. This means that a much smaller number of neurons is required than is the case for fully connected networks, which results in considerable reduction in computing time and memory requirements. However, it is only in recent years that it has become possible to develop networks that can handle huge amount of input data for the detection of more complex objects. This is due in particular to the availability of powerful and highly parallel graphics processors (graphical processing units, GPUs) [26]. CNNs have played a major role in the success of DL for image analysis in a wide range of applications including image classification [26, 33, 34], image segmentation [34], instance segmentation [34] and object detection $[35,36]$.
Image classification The breakthrough for such deep networks was achieved in 2012 with AlexNet [26]. This network achieved much better results for the classification of images from a benchmark data set than all classical methods for object recognition. The schematic structure of a network for classification is shown in the upper part of Fig. 2. Clearly visible is the reduction of the resolution with increasing depth of the network, with simultaneous increase of the feature space. By reducing the resolution at the transition between the layers of the network, an ever larger area of the image is also considered. In the end, there is usually an analysis of the entire image by several completely connected layers. The weights of the neurons are calculated during the training of the network using manually annotated training data. The weights are optimized by backpropagation, a special variant of the gradient descent method. At the end, the weights contain all the information necessary for the classification of objects. They decide how strongly a characteristic represented by a filter is weighted and how much neurons in subsequent layers are activated.

Image segmentation CNNs are not only suitable for classifying an entire image (one class per image), but also for the challenging task of semantic segmentation. Such networks have a much more complex task, namely the classification of each pixel in an image, as compared to networks for image classification, where the output is to determine the probability of image belonging to a certain class. An architecture that has been proposed for this task is the so-called fully convolutional networks (FCN) [37]. These networks add further layers to the classification part, which upsample the feature maps to achieve segmentation maps that have the same resolution as the input data. An example of such an encoder-decoder network architecture is the U-Net [8], which is successfully used for segmenting biomedical images. U-Net is a simple but powerful network, it is fast to train and delivers precise segmentation $[8,38]$. It requires fewer amount of data to achieve good results, while retaining the possibility to train on large sample sizes [39]. Since its introduction, it has achieved top ranks in various medical imaging tasks and its architecture is used as a basis in many DL models [38-44]. The encoder part of the U-Net network applies convolution and max-pooling operations to extract useful semantic information from images. The decoder part upsamples this information with the help of spatial information acquired using skip connections from higher-resolution feature maps in the encoder part [42]. The result is a precise segmentation, in other words per pixel classification, of structures in the given image. Figure 3 shows a schematic overview of this network architecture.

Instance segmentation A segmentation approach that combines object detection [35] and semantic segmentation [37], the so-called instance segmentation, is a rapidly growing application area of CNNs. The idea is to localize objects 

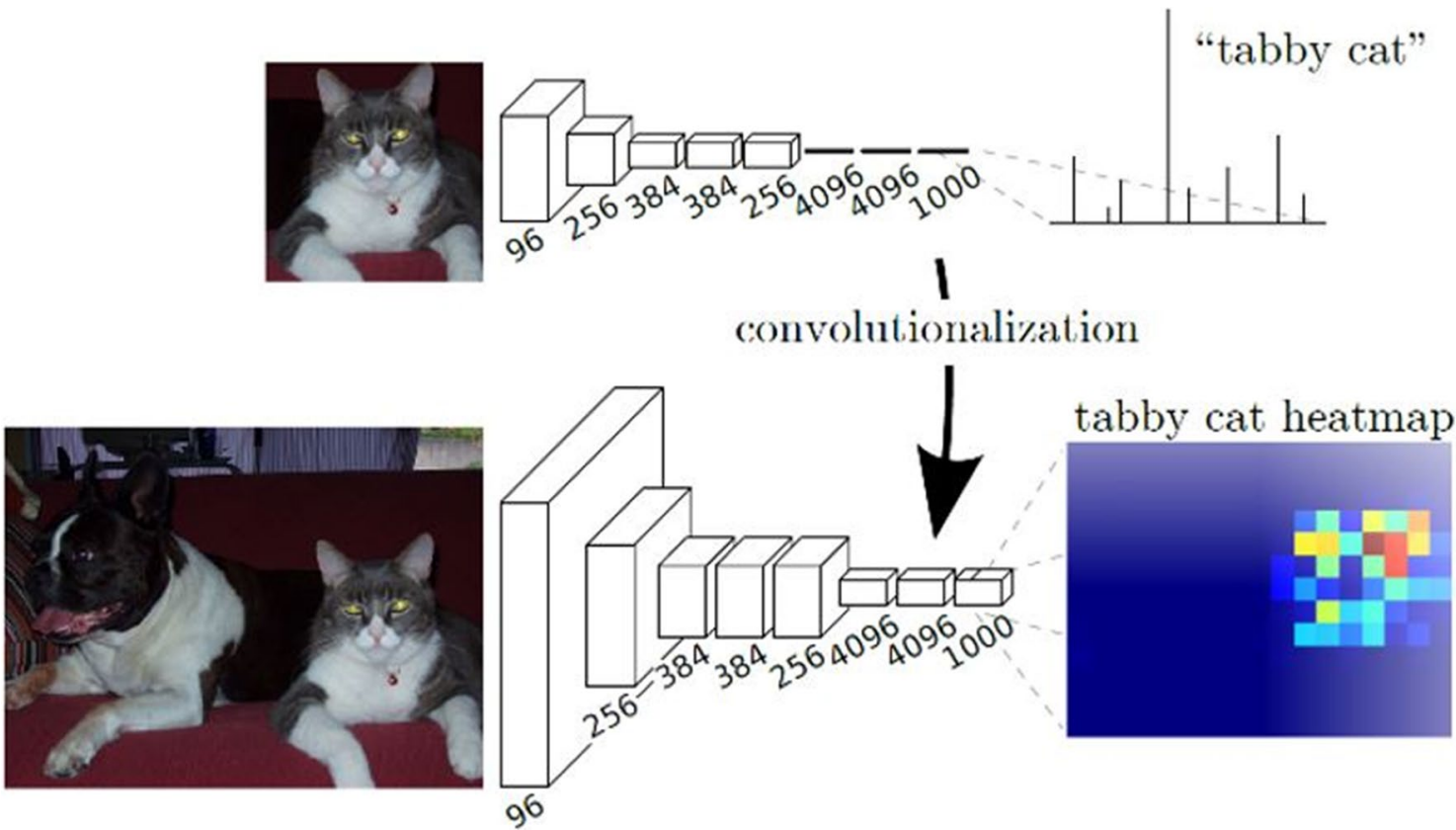

Fig. 2 Schematic comparison of the architecture of two neural networks. Above: network for classification; below: network for calculating the approximate localization of an object using the activations of the feature map (heat map). The numbers below the layers represent the number of feature maps (Source: [29])

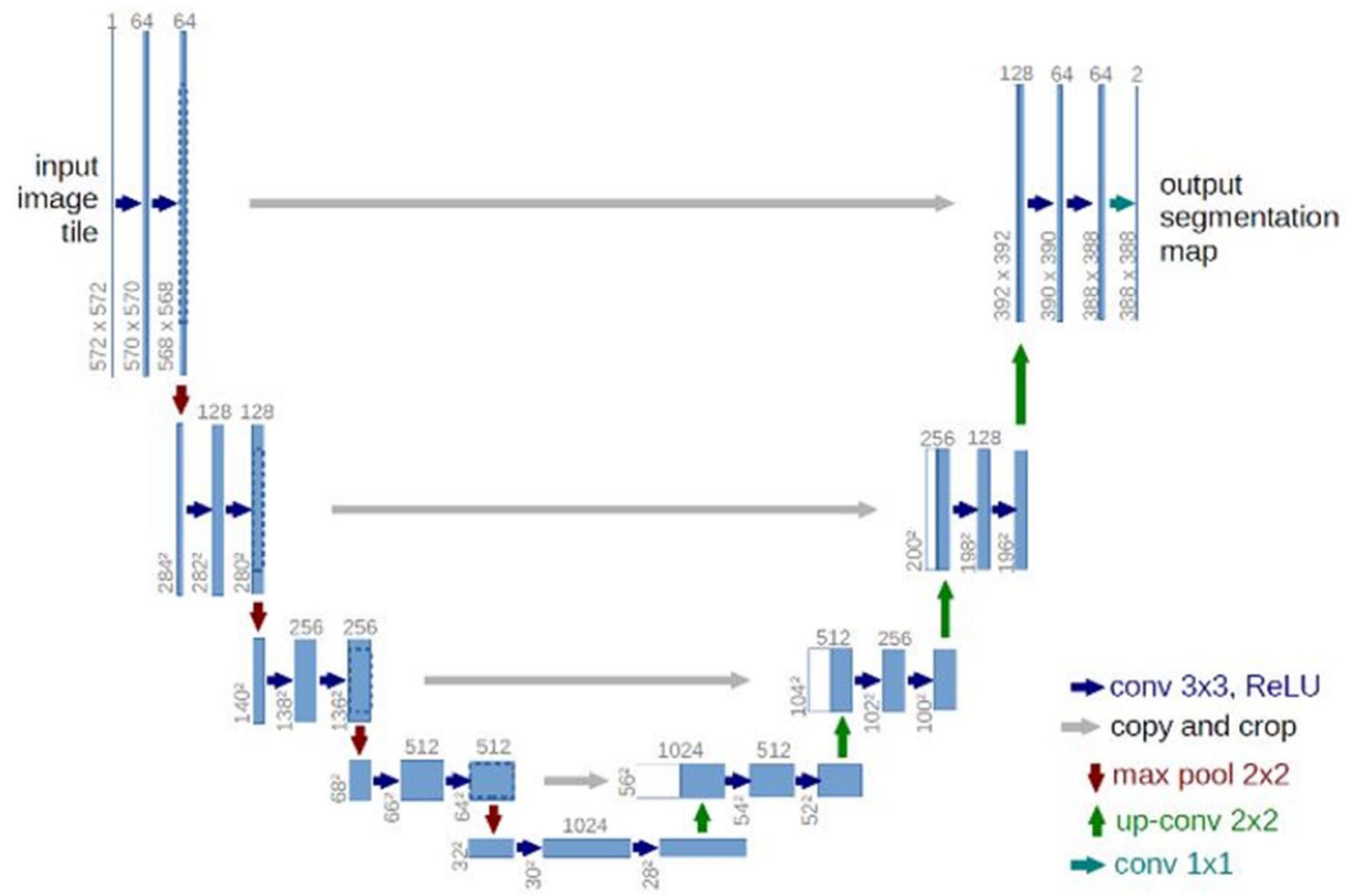

Fig. 3 Schematic view of the architecture of an up-convolutional network for the semantic segmentation of images. The left part takes over the classification, while the information about the localization of objects is lost. The right part restores this information by upsampling (Source: [9]) 
in an image and precisely segment each instance [45]. For medical image analysis, instance segmentation models such as Mask-RCNN are reported to be powerful at detecting instances accurately, but generate less accurate segmentation masks compared to U-Net [46].

An essential requirement for training neural networks is to have a sufficient amount of annotated training data. Manual annotation is time-consuming; for instance, in image segmentation each object must be traced exactly. Ideally, the training data and the test data should come from the same distribution. While it is possible to classify objects in similar scenes with networks pre-trained on data from related domains, a good quality training data is very crucial. Training on large amount of data is very computation intensive and can take several days to weeks even on powerful graphics cards [47, 48]. During inference, however, the fully trained networks usually carry out the analysis of an image quickly. Typical values are processing times from $5 \mathrm{~ms}$ per image for simple tasks up to approximately $500 \mathrm{~ms}$ for more complex analyses using a current GPU; of course, this depends on the degree of optimization of the algorithms, training procedures, and the performance of the hardware used $[35,47]$.

Another challenge in training DL models is their sensitivity to many hyperparameters that have to be set properly to achieve optimal results. Tuning these hyperparameters manually requires domain knowledge of their effect on the generalization of the network [32] and is time intensive. Significant progress has been done in automatic tuning of hyperparameters for DL models; however, the process is still computationally intensive [49-52].

A clear advantage of neural networks is the possibility of end-to-end training and the fact that neural networks are very powerful at generalizing to never seen data. Exemplary results of two current approaches to semantic segmentation can be seen in Fig. 4.

\section{Application of ANNs for urological endoscopy}

The field of urology has benefitted from recent advances in DL. However, in early stages, significant work has been done toward application of DL in areas such as image classification [26], object detection [35] and image segmentation. Ikeda et al. trained a CNN with 177 images of histologically confirmed bladder tumors and 133 images of healthy urothelium [53]. The neural network was pre-trained with 1.2 million images from ImageNet data set [54], which were classified into 1000 categories. The output revealed $93.0 \%$ sensitivity and $83.7 \%$ specificity for differentiating between tumoral and healthy urothelium.

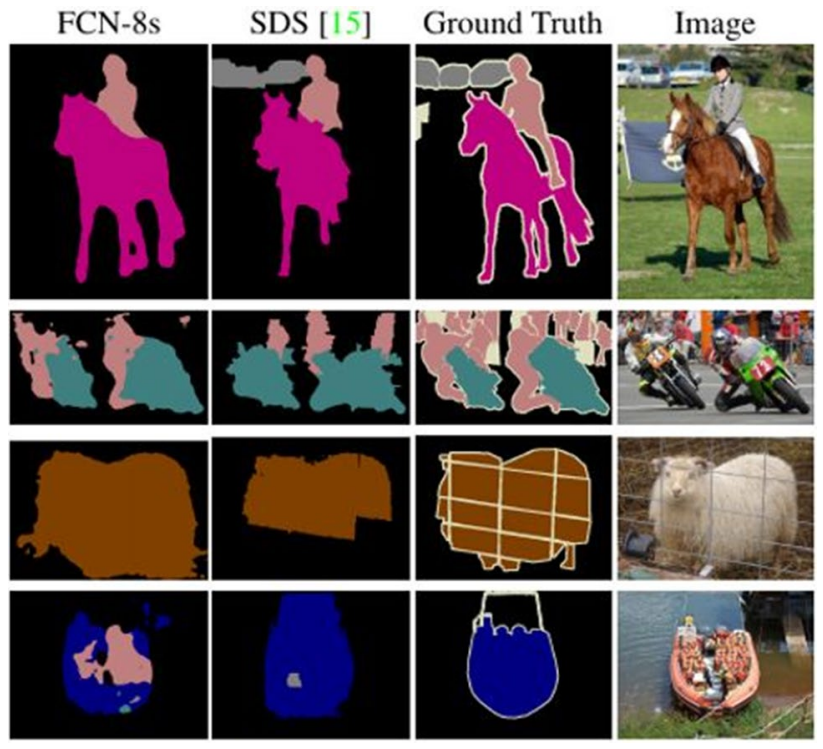

Fig. 4 Semantic segmentation divides an image into semantically related regions by assigning each pixel of an image to a specific class (coded here by color). Ground truth is the manual annotation. The segmentation of complex scenes is still a challenge (Source: [31])

Eminaga et al. studied 479 cystoscopy videos from patients with 44 urologic findings [6]. The authors used data augmentation to enlarge their data set of 479 images by up to a factor of 40 , resulting in 18,681 images. The authors evaluated several CNN models and found that the Xception-based model achieved the highest accuracy score $(99.52 \%)$ for the diagnostic classification of cystoscopic images. Nonetheless, $7.86 \%$ of bladder stone images and $1.43 \%$ containing indwelling catheters were falsely classified [6].

In a recent work, on automated detection of $\mathrm{BCa}$ on cystoscopic images, Shkolyar et al. used deep CNNs to detect suspicious lesions from cystoscopic videos [1]. The study uses TUMNet, an image analysis software based on DL to detect bladder papillary tumors on cystoscopic videos of 100 patients. The software first extracts video frames containing tumors and segments tumors within the selected frames. The authors report $90 \%$ sensitivity for tumor detection. Table 1 summarizes the current AI approaches in cystoscopic image analysis.

The impact of $\mathrm{AI}$ in interpreting cystoscopic images is currently under study in a recent collaborative research project, RaVeNNA-4pi. The goal of the project is to develop a digital platform with 4PI Endoimaging for 3D-reconstruction, semantic segmentation and visualization of the bladder for $\mathrm{BCa}$ documentation and improved patient follow-up.

RaVeNNA-4pi focuses on multi-class segmentation of cystoscopic images using DL. Semantic segmentation allows precise segmentation of classes of interest such as tumors in an image and is helpful in documentation and visualization of tumors in a 3D-reconstructed bladder. As 
Table 1 Summary of current approaches of AI in cystoscopic images

\begin{tabular}{|c|c|c|c|}
\hline Author & Training procedure & Data set & Prediction accuracy \\
\hline \multicolumn{4}{|c|}{ Artificial neural networks on analysis of cystoscopic images } \\
\hline Eminaga et al. 2018 [6] & Image classification, 44 classes & $\begin{array}{l}\text { 18, } 681 \text { training images, used data } \\
\text { augmentation }\end{array}$ & $\begin{array}{l}\text { Xception model: F1 score ( } 99.52 \%) \text {. } \\
\text { Other models } 0.04-4.05 \text { difference } \\
\text { in performance to Xception model }\end{array}$ \\
\hline Shkolyar et al. 2015 [1] & $\begin{array}{l}\text { Instance segmentation, } 2 \text { classes: } \\
\text { cancer and benign }\end{array}$ & $\begin{array}{l}\text { Training with } 417 \text { cancer and } 2335 \\
\text { normal frames, validation } 211 \\
\text { cancer, } 1002 \text { normal frames }\end{array}$ & $\begin{array}{l}\text { per-frame sensitivity was } 88 \% \text {, } \\
\text { per-frame specificity was } 99 \% \text {, per- } \\
\text { tumor sensitivity was } 90 \%\end{array}$ \\
\hline Ikeda et al. 2018 [53] & $\begin{array}{l}\text { Image classification, binary classes: } \\
\text { tumoral and healthy urothelium }\end{array}$ & $\begin{array}{l}\text { Data from } 177 \text { tumor lesion images } \\
\text { and } 133 \text { normal images }\end{array}$ & $\begin{array}{l}93.0 \% \text { sensitivity and } 83.7 \% \text { specific- } \\
\text { ity }\end{array}$ \\
\hline
\end{tabular}

part of the project, a novel data set of cystoscopic images was created and annotated by trained investigators. For the data acquisition process, patients' cystoscopy videos were collected from cystourethroscopy procedures. These procedures were performed with a rigid cystoscope and a $30^{\circ}$ lens under white light. Video frames of interest were extracted and manually annotated using a special label editor software (developed at the Fraunhofer Institute for Physical Measurement Techniques IPM). The annotation was conducted by two physicians and subsequently confirmed by a board-certified urologist. Classes of interest in this project are: (1) papillary tumor, (2) carcinoma in situ suspicion, (3) resected tumor, (4) bladder diverticulum, (5) bladder stone, and (6) left and right ureteric orifices. The RaVeNNA cystoscopy data set currently consists of 4200 annotated images extracted from 62 videos. An example of images is shown in Fig. 5.

The procedure uses U-Net, an encoder-decoder network that has been used successfully in various medical image analysis tasks $[8,38,42]$. The network architecture was slightly modified by adding batch normalization after each convolutional layer. To tackle class imbalance in the data, we use a weighted categorical cross-entropy loss. Data augmentation methods such as image rotation and zoom were used to make the network more robust.

For training purposes, $70 \%$ of the data were used for training, $10 \%$ for validation, and $20 \%$ for test. The network achieved 0.67 average dice score coefficient (DSC). Examples of original images, annotation and automated segmentations are shown in Fig. 6. The study shows that deep CNNs can be used for semantic segmentation of multiple cystoscopic image categories.

We found that certain classes, such as papillary tumors and Cis suspicion, have high inter-class similarities and therefore are challenging for pixel-wise classification, and thereby degrade the average DSC performance over all classes. The current research work focuses on developing methods to distinguish these classes with higher precision/ recall.

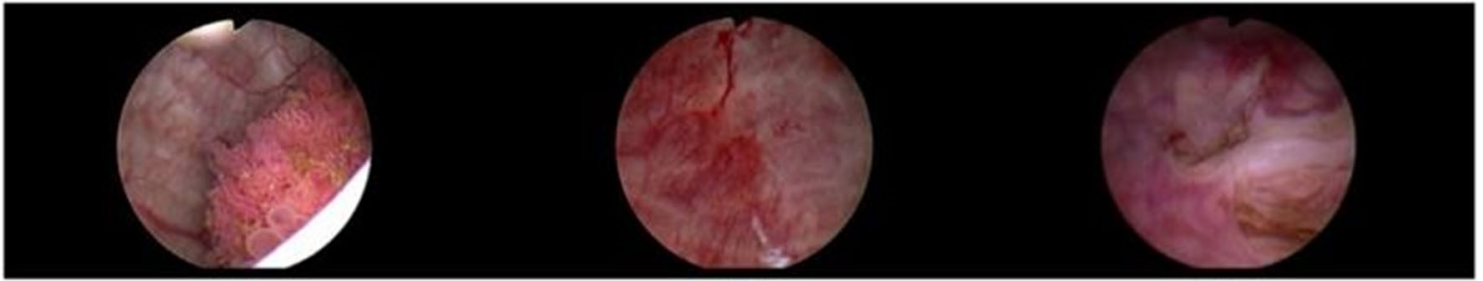

(1)

(2)

(3)

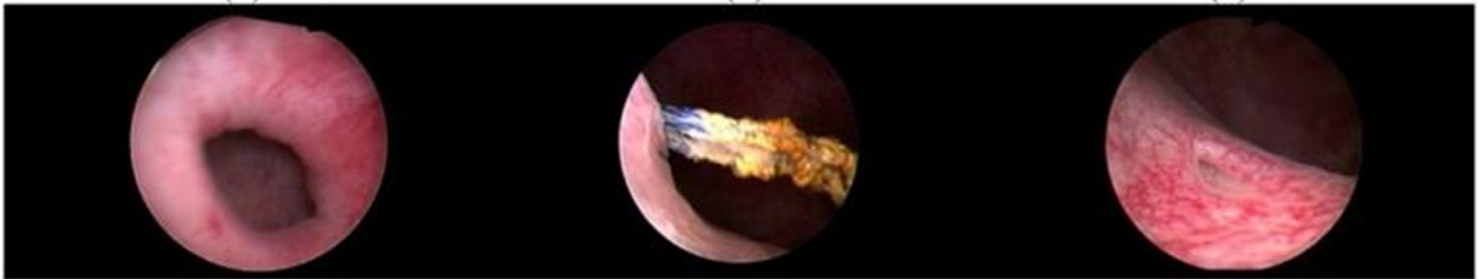

(4)

(6)

Fig. 5 Representative images from RaVeNNA cystoscopy data set. From top left to down right, we show the classes: (1) papillary tumor, (2) ca in situ suspicion, (3) resented tumor, (4) diverticulum, (5) bladder stone, and (6) left and right ureteric orifice 

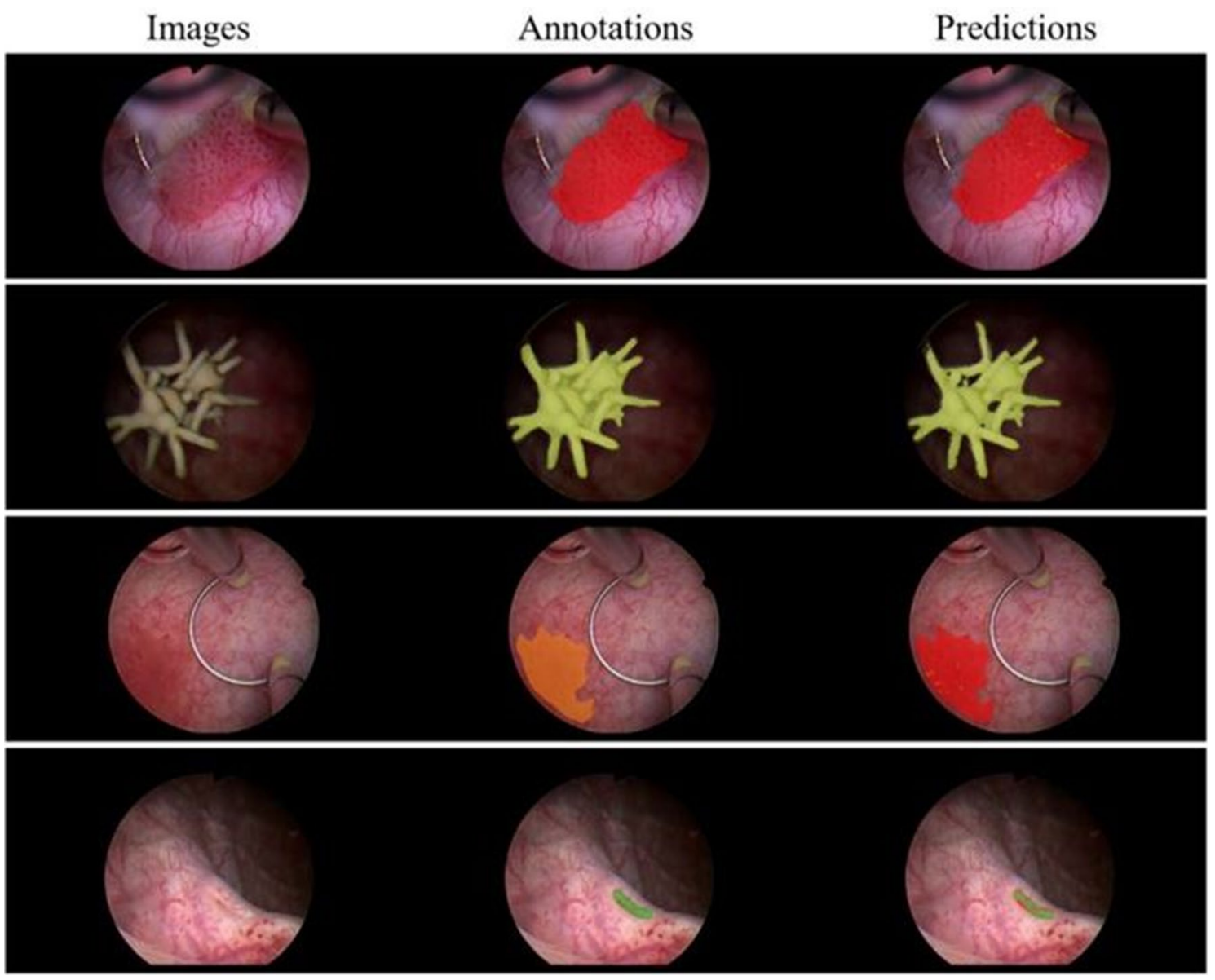

Fig. 6 Representative images show results of the network. Each row shows an image from a specific class-papillary tumor, bladder stone, ca in situ and orifice-from top to bottom, respectively. On the far left are images, in the middle the manual annotations and on the right

\section{Conclusion}

Medical care is one of the most important areas of a modern society. The contributions made in this field in clinics, research and teaching have continuously improved the standard of living of citizens and increased life expectancy worldwide. In the recent past, medical research has been strongly influenced by the inclusion of IT methods and different microsystem technologies among diagnostic and therapeutic means. Machine learning (ML) enables highly efficient investigation of large data sets in which regularities and patterns are searched for. Particularly in cystoscopy, image-based data are generated that have not yet been adequately researched and exploited. In this work, outlines and potential of these technologies for application in urology were discussed. The next years will certainly be shaped by the incorporation of AI methods into result interpretation and reporting by endoscopic examinations. Scientific activities in this field should therefore be intensified. predictions of the network. The image in the third row is an example of how a papillary tumor and a bladder lesion suspicious for ca in situ can be misclassified

Acknowledgements Open Access funding provided by Projekt DEAL. The authors thank Grigor Andreev and Manuel Hecht for their valuable work in data annotation.

Author contributions $\mathrm{MN}$ : data management, data analysis and interpretation, manuscript writing/editing. SH: manuscript writing/editing. RSI: data collection, manuscript writing/editing. AM: project development, data and result evaluation, manuscript writing/editing. AR: project development, data and result evaluation, manuscript writing/ editing.

Funding This study was funded by the German Federal Ministry of Education and Research (grant number 13GW0203A).

\section{Compliance with ethical standards}

Conflict of interest The authors declare that they have no conflict of interest.

Ethical approval All procedures performed in this study were approved by the local Ethical Committee of the University of Freiburg, Germany. 
This article does not contain any studies with animals performed by any of the authors.

Informed consent Subject retrospective data was handled with anonymity; therefore, no informed consent was obtained from all individual participants included in the study.

Open Access This article is licensed under a Creative Commons Attribution 4.0 International License, which permits use, sharing, adaptation, distribution and reproduction in any medium or format, as long as you give appropriate credit to the original author(s) and the source, provide a link to the Creative Commons licence, and indicate if changes were made. The images or other third party material in this article are included in the article's Creative Commons licence, unless indicated otherwise in a credit line to the material. If material is not included in the article's Creative Commons licence and your intended use is not permitted by statutory regulation or exceeds the permitted use, you will need to obtain permission directly from the copyright holder. To view a copy of this licence, visit http://creativecommons.org/licenses/by/4.0/.

\section{References}

1. Boslaugh SE (2007) American cancer society. In: Colditz G (ed) Encyclopedia of cancer and society. SAGE Publications Inc, Thousand Oaks.

2. Siegel RL, Miller KD (2019) Jemal A (2019) Cancer statistics. CA Cancer J Clin 69(1):7-34. https://doi.org/10.3322/caac.21551

3. Robert Koch Institute Cancer in Germany 2013/2014 2018 (German Centre for Cancer Registry Data 11th Edition)

4. Freitas NR, Vieira PM, Lima E et al (2017) Using cystoscopy to segment bladder tumors with a multivariate approach in different color spaces. Conf Proc IEEE Eng Med Biol Soc 2017:656-659. https://doi.org/10.1109/EMBC.2017.8036910

5. Babjuk M, Böhle A, Burger M et al (2017) EAU guidelines on non-muscle-invasive urothelial carcinoma of the bladder: update 2016. Eur Urol 71(3):447-461

6. Eminaga O, Eminaga N, Semjonow A et al (2018) Diagnostic classification of cystoscopic images using deep convolutional neural networks. JCO Clin Cancer Inform 2:1-8. https://doi.org/10.1200/ CCI.17.00126

7. Freitas NR, Vieira PM, Lima E et al (2018) Automatic T1 bladder tumor detection by using wavelet analysis in cystoscopy images. Phys Med Biol 63(3):35031. https://doi.org/10.1088/1361-6560/ aaa3af

8. Ronneberger OA, Fischer A, Philipp A, Brox T (2015) U-net: convolutional networks for biomedical image segmentation. Springer, Berlin

9. Wu L, Zhang J, Zhou W et al (2019) Randomised controlled trial of WISENSE, a real-time quality improving system for monitoring blind spots during esophagogastroduodenoscopy. Gut. https ://doi.org/10.1136/gutjnl-2018-317366

10. Wang P, Berzin TM, Brown JR et al (2019) Real-time automatic detection system increases colonoscopic polyp and adenoma detection rates: a prospective randomised controlled study. Gut. https://doi.org/10.1136/gutjnl-2018-317500

11. Rees CJ, Koo S (2019) Artificial intelligence-upping the game in gastrointestinal endoscopy? Nat Rev Gastroenterol Hepatol. https ://doi.org/10.1038/s41575-019-0178-y

12. Fradet $Y$, Grossman HB, Gomella L et al (2007) A comparison of hexaminolevulinate fluorescence cystoscopy and white light cystoscopy for the detection of carcinoma in situ in patients with bladder cancer: a phase III, multicenter study. J Urol 178(1):6873. https://doi.org/10.1016/j.juro.2007.03.028
13. Hermann GG, Mogensen K, Carlsson S et al (2011) Fluorescenceguided transurethral resection of bladder tumours reduces bladder tumour recurrence due to less residual tumour tissue in $\mathrm{Ta} /$ T1 patients: a randomized two-centre study. BJU Int 108(8 Pt 2):E297-303. https://doi.org/10.1111/j.1464-410X.2011.10090.x

14. Daneshmand $\mathrm{S}$ et al (2018) Blue light cystoscopy for the diagnosis of bladder cancer: Results from the US prospective multicenter registry. Urol Oncol 36(8):361

15. Burger M, Grossman HB, Droller M et al (2013) Photodynamic diagnosis of non-muscle-invasive bladder cancer with hexaminolevulinate cystoscopy: a meta-analysis of detection and recurrence based on raw data. Eur Urol 64(5):846-854. https://doi. org/10.1016/j.eururo.2013.03.059

16. Babjuk M, Burger M, Comperat E et al (2018) EAU guidelines on non-muscle-invasive bladder cancer (TaT1 and CIS). European Association of Urology

17. Kang W, Cui Z, Chen Q et al (2017) Narrow band imagingassisted transurethral resection reduces the recurrence risk of non-muscle invasive bladder cancer: a systematic review and meta-analysis. Oncotarget 8(14):23880-23890. https://doi. org/10.18632/oncotarget.13054

18. Chen SP, Liao JC (2014) Confocal laser endomicroscopy of bladder and upper tract urothelial carcinoma: a new era of optical diagnosis? Curr Urol Rep 15(9):437. https://doi.org/10.1007/ s11934-014-0437-y

19. Raharja PAR, Hamid ARAH, Mochtar CA et al (2018) Recent advances in optical imaging technologies for the detection of bladder cancer. Photodiagnosis Photodyn Ther 24:192-197. https://doi.org/10.1016/j.pdpdt.2018.10.009

20. Tully K, Palisaar R-J, Brock M et al (2019) Transurethral resection of bladder tumours: established and new methods of tumour visualisation. Transl Androl Urol 8(1):25-33. https:// doi.org/10.21037/tau.2018.12.12

21. Huang J, Ma X, Zhang L et al (2018) Diagnostic accuracy of optical coherence tomography in bladder cancer patients: a systematic review and meta-analysis. Mol Clin Oncol 8(4):609612. https://doi.org/10.3892/mco.2018.1566

22. Chen H, Li X, Broderick $\mathrm{N}$ et al (2018) Identification and characterization of bladder cancer by low-resolution fiber-optic Raman spectroscopy. J Biophoton 11(9):e201800016. https:// doi.org/10.1002/jbio.201800016

23. Kolenc OI, Quinn KP (2019) Evaluating cell metabolism through autofluorescence imaging of $\mathrm{NAD}(\mathrm{P}) \mathrm{H}$ and FAD. Antioxid Redox Signal 30(6):875-889. https://doi.org/10.1089/ ars.2017.7451

24. Pradère B, Poulon F, Compérat E et al (2018) Two-photon optical imaging, spectral and fluorescence lifetime analysis to discriminate urothelial carcinoma grades. J Biophoton 11(11):e201800065. https://doi.org/10.1002/jbio.201800065

25. Lowe DG (2004) Distinctive image features from scale-invariant keypoints

26. Krizhevsky A, Sutskever I, Hinton GE (2012) ImageNet classification with deep convolutional neural networks, pp 1097-1105

27. Lecun Y, Bottou L, Bengio Y, Haffner P (1998) Gradient-based learning applied to document recognition

28. Bishop CM (2006) Pattern recognition and machine learning (Information Science and Statistics)

29. Rumelhart DE, Hinton GE, Williams RJ (1986) Learning representations by back-propagating errors. Nature 323(6088):533. https://doi.org/10.1038/323533a0

30. Cybenko G (1989) Approximation by superpositions of a sigmoidal function. Math Control Signals Syst 2(4):303-314. https://doi. org/10.1007/BF02551274

31. Zeiler MD, Fergus R (2013) Visualizing and understanding convolutional networks. https://arxiv.org/pdf/1311.2901 
32. Goodfellow I, Bengio Y, Courville A (2016) Deep learning. MIT Press, Cambridge, Massachusetts, London, England

33. Simonyan K, Zisserman A (2015) Very deep convolutional networks for large-scale image recognition. https://arxiv.org/ pdf/1409.1556

34. He K, Zhang X, Ren S et al (2015) Deep residual learning for image recognition. https://arxiv.org/pdf/1512.03385

35. Ren S, He K, Girshick R et al. (2015) Faster R-CNN: towards real-time object detection with region proposal networks

36. Uijlings JRR, van de Sande KEA, Gevers T et al (2013) Selective search for object recognition. Int J Comput Vis. https://doi. org/10.1007/s11263-013-0620-5

37. Long J, Shelhamer E, Darrell T (2014) Fully convolutional networks for semantic segmentation

38. Çiçek Ö, Abdulkadir A, Lienkamp SS et al. (2016) 3D U-net: learning dense volumetric segmentation from sparse annotation. https://arxiv.org/pdf/1606.06650

39. Falk T, Mai D, Bensch R et al (2019) U-Net: deep learning for cell counting, detection, and morphometry. Nat Methods 16(1):67-70. https://doi.org/10.1038/s41592-018-0261-2

40. Oktay O, Schlemper J, Le Folgoc L et al (2018) Attention U-Net: learning where to look for the pancreas

41. Chen W, Liu B, Peng S et al (2019) S3D-UNet: separable 3D U-Net for brain tumor segmentation. In: Crimi A (ed) Brain lesion: glioma, multiple sclerosis, stroke and traumatic brain injuries: 4th international workshop, BrainLes 2018, held in conjunction with MICCAI 2018, Granada, Spain, September 16, 2018, Revised Selected Papers, vol 11384. Springer, Cham, Switzerland, pp 358-368

42. Isensee F, Petersen J, Klein A et al. (2018) nnU-net: self-adapting framework for U-net-based medical image segmentation

43. Gordienko Y, Gang P, Hui J et al (2019) Deep learning with lung segmentation and bone shadow exclusion techniques for chest X-ray analysis of lung cancer 754(1): 638-647. doi: 10.1007/978-3-319-91008-6_63

44. Ma X, Hadjiiski L, Wei J et al (2019) 2D and 3D bladder segmentation using U-Net-based deep-learning. In: International society for optics and photonics, 109500Y
45. He K, Gkioxari G, Dollár P et al (2018) Mask R-CNN. https:// arxiv.org/pdf/1703.06870

46. Vuola AO, Akram SU, Kannala J (2019) Mask-RCNN and U-net ensembled for nuclei segmentation. https://arxiv.org/ pdf/1901.10170

47. Coleman CA, Narayanan D, Kang D et al (2017) DAWNBench: an end-to-end deep learning benchmark and competition

48. Chen L-C, Papandreou G, Kokkinos I et al (2018) DeepLab: semantic image segmentation with deep convolutional nets, atrous convolution, and fully connected CRFs. IEEE Trans Pattern Anal Mach Intell 40(4):834-848. https://doi.org/10.1109/TPAMI .2017.2699184

49. Falkner S, Klein A, Hutter F (2018) BOHB: robust and efficient hyperparameter optimization at scale

50. Domhan T, Springenberg JT, Hutter F (2015) Speeding up automatic hyperparameter optimization of deep neural networks by extrapolation of learning curves

51. Ilievski I, Akhtar T, Feng J et al (2016) Efficient hyperparameter optimization of deep learning algorithms using deterministic RBF surrogates

52. Li L, Jamieson KG, DeSalvo G et al (2017) Hyperband: banditbased configuration evaluation for hyperparameter optimization. ICLR

53. Ikeda A, Hoshino Y, Nosato $\mathrm{H}$ et al (2018) Objective evaluation for the cystoscopic diagnosis of bladder cancer using artificial intelligence. Eur Urol Suppl 17(2):e1230-e1231. https://doi. org/10.1016/S1569-9056(18)31702-0

54. Deng J, Dong W, Socher R, Li LJ, Li K, Fei-fei L (2009) Imagenet: a large-scale hierarchical image database

55. Shkolyar E, Jia X, Xing L et al (2019) Automated cystoscopic detection of bladder cancer using deep-Learning. J Urol. https:// doi.org/10.1097/01.JU.0000557512.76700.42

Publisher's Note Springer Nature remains neutral with regard to jurisdictional claims in published maps and institutional affiliations. 